\title{
FROM SCIENCE STUDENT TO SCIENTIST
}

\author{
DEREK S. WANG* \& DAVID D. DAI
}

October 2020

\begin{abstract}
Science students are taught that science is a collection of facts and equations when in fact, science is a journey full of false starts, dead ends, and creative detours undertaken by scientists to uncover the truth of reality. A science student seeking to become a scientist must often regress back to a state of childlike wonder and curiosity to prepare for such a journey. We seek to spark this change with hundreds of Quick Takes and Inquiries into specifically chemistry and physics at the introductory level. Each question demands the student to strike out into the wilderness of knowledge - to think deeply, read and ask widely, and model extensively. Upon completion of this curriculum, students should be equipped with the knowledge and mindset to conceive of and execute independent scientific investigations of their own. We also hope that science teachers are inspired to incorporate our open-ended investigations into their own curricula.
\end{abstract}

* Harvard University, Cambridge, MA o2138, USA

$\dagger$ The Harker School, San Jose, CA 95129, USA 


\section{CONTENTS}

Preface

1 The Quantum World

2 Quantum Mechanics in Action: Atoms

3 Chemical Bonds

4 Molecular Shape and Structure

6 Liquids and Solids

7 Inorganic Materials

8 Thermodynamics: The First Law

9 Thermodynamics: The Second and Third Laws

10 Physical Equilibrium

11 Chemical Equilibria

12 Acids and Bases

13 Aqueous Equilibria

14 Electrochemistry

15 Chemical Kinetics

16 The Elements: The Main-Group Elements

17 The Elements: The d-block 


\section{PREFACE: TO THE BUDDING SCIENTIST}

How do a science student and scientist differ? Simply put, science students are posed well-defined problems and expected to parrot concrete solutions, while scientists must 1) distill their own research questions and 2) respond in kind with no step-by-step map. To become a scientist, the student must learn to both ask the right questions and answer them through whatever means necessary. Perhaps surprisingly, this transition is not a progression, but rather a regression to the state of pure curiosity that we all embodied as children. We plopped into this big, formidable world with no conception for the rules, where the only way to learn more was to ask questions and propose solutions, e.g. "How do we get mom and dad to give us food? Let's try crying, laughing, rolling, sleeping, or any combination thereof."

For many students nearing the end of high school or college, their natural curiosity and motivation to satiate it has long since dissipated. We believe the reason for this unfortunate transition is that students in typical classroom settings, instead of generating questions and untangling the solutions themselves, are given both without fail. Coupled with the need to succeed under time constraints, the provided questions and methods form a boundary around what "needs" to be known and what is extraneous. But for every question asked on an exam, there are a hundred more readily available that could have been asked, a thousand more that haven't even been conceived of yet, and ten thousand ways of approaching each one.

With the following questions, we are pushing you to distill the most critical questions in response and solve them through whatever means necessary, to become a scientist while still a science student. The questions we pose spring directly from textbook material—Chemical Principles: The Quest for Insight, 6th Edition by Peter Atkins, Loretta Jones, and Leroy Laverman, an introductory chemistry textbookand are written for particularly curious and clever students who want to leap beyond appreciating the wonders of nature to actively taking part in unraveling them. They are left intentionally vague and broad so that you will have to generate and prioritize sub-questions and sub-sub-questions. In a sense, you will construct a mini-research study for each question. Ideally, they will initiate a self-propagating loop throughout your career of solving one problem only to discover many more along the way.

Perhaps if you are lucky, you may apply a method you are already familiar with, but more often than not, you will have to invent new methodologies, read extensively, discuss with colleagues and teachers, try something and fail countless times, and more. You're likely not comfortable doing this, as typical classroom settings present a single method as the be-all and end-all for a given problem. And if you have been taught under this model for a decade or more of your life, how can you be expected to suddenly think so differently when pursuing your first research project? To further motivate you to think deeply, we point out important applications in quantum computing, drug development, and more; discuss open problems that researchers continue to toil over; draw parallels between material you may have learned elsewhere; and suggest further readings, textbooks, resources, and experiments to try.

Concretely, regarding the content of this document, "Quick Takes" are shorter and simpler, serving as warm-ups to the "Inquiries," which are more difficult and may require synthesizing multiple approaches. For instance, some questions are best solved by deriving an equation, while others ask you to pull knowledge from adjacent fields (i.e. physics), while others need only a hand-wavey qualitative explanation. You may need to consult literature-typically Wikipedia is fine, but we may refer you to other textbooks-or you may discuss them with friends and teachers. Ultimately, you will have to decide how to approach each question. Of course, we won't just leave you in the dust. Typically, you will be able to make decent progress using language and formalisms you will become familiar with by simply reading 
the textbook beforehand, although to get better and more comprehensive solutions, you will have to venture onto unfamiliar ground, just as scientists do.

Finally, we are confident that a student with a basic knowledge of physics and calculus can learn the material necessary to make a dent in each one of these questions. After all, one of the co-authors of this document was the original audience for these questions and did exactly that!

Let's take a step backwards, re-spark our curiosity, and unravel the wonders of nature.

DeReK S. WANG and David. D. DAI, October 2020 


\section{Quick Takes}

1. Wave function units What are the units of a quantum wave function?

2. Atomic Hamiltonian What would a Hamiltonian (and if you don't know what a Hamiltonian is, for now you just need to know that it is an equation for the total energy of a system) for a generic atom look like? You'll find a specific term in this Hamiltonian that is responsible for the unfortunate impossibility of exactly calculating the energy of an atom.

3. Wave functions are "complex" A wave function as a function of space is often a complex function (i.e. has a real and complex component for a given position r)-what does it mean for the wave function to be complex? Explain why the phase factor of a wave function (mostly) does not affect what we observe, such as probability or energy. ${ }^{1}$

\section{Inquiries}

1. Photon wavenumber What does it mean when we say "a photon with wavenumber $\mathbf{k}$ is emitted"? Are all photons with the same frequency identical? Typically, we think of photons as short pulses of light with a specific frequency. How does one reconcile this with the Fourier/Heisenberg uncertainty principle, which states that simultaneous wavenumber and space localization is impossible?

2. Photon wavefunction Since a photon can be thought of as a quantum particle, what does its wave function look like or correspond to? Given a "photonic wavefunction", how would we calculate the resulting electromagnetic field? Is a photonic wavefunction even well-defined?

3. Identical particles For a system of $\mathrm{N}$ identical quantum particles, we will have wavefunction $\psi\left(r_{1}, r_{2}, \ldots, r_{N}\right)$, where $r_{i}$ is the coordinate vector for particle $i \in\{1, N\}$. Because the quantum particles are indistinguishable, the wavefunction should have some symmetry under permutation of its arguments. Can you determine this symmetry constraint? Can this symmetry constraint be used to classify particles? ${ }^{2}$

4. Variational principle In classical physics, when a system is in static equilibrium its energy is minimized (this is where the principle of virtual work comes from). Can you find a similar principle in quantum mechanics?

5. Spin Magnetic Moment How could we design an experiment to test whether an electron spin really corresponds to a magnetic moment? How could we measure the strength of the magnetic moment?

Concluding Notes "Photon wavenumber" and "Photon wavefunction" are among the questions that Feynman wrestled with that led him (and a few others) to develop quantum electrodynamics (QED). It turns out, as you should deduce yourself, that quantum mechanics is not a sufficient theory to explain the quantum nature of light. What you need is a field theory, where photons can pop in and out of existence as excitations of a quantized field. If you are interested in learning a little more, check out QED: The Strange Theory of Light and Matter, a series of four lectures by Feynman meant to be understood by the general public. There, you'll get a sense for further contradictions between quantum mechanics and classical mechanics and the "true" nature of light.

I In fact, the phase factor can be observed under carefully designed experiments. See an introduction to Berry phases.

2 When you're done, look up anyons. 


\section{QUANTUM MECHANICS IN ACTION: ATOMS}

\section{Quick Takes}

1. Particle vs wave An electron is more easily visualized as a particle, especially within an atom, but sometimes one uses the "wave" description when explaining effects like electron diffraction. In solids, it's typically more helpful to visualize an electron as a wave. Give a hand-wavey explanation for why.

2. Lattice defects What would the orbitals around a single vacancy in a solid look like? Explain why they might be expected to emit photons upon excitation, just like a hydrogen atom would.

3. Relativistic electrons The Schrodinger equation assumes non-relativistic electrons, which is an approximation. In the hydrogen atom, is this reasonable? At what atomic number might this approximation break down?

\section{Inquiries}

1. Delta bond A single bond is when the lobes of orbitals (typically s orbitals) of two atoms constructively overlap, and a pi bond is when two lobes of orbitals (typically p orbitals) of each of two atoms align but do not directly overlap constructively-what would bonding between two d orbitals look like? Don't forget to consider that there are 5 of them, each with different $m_{l}$.

2. Non-interacting electrons Suppose, in an imaginary universe, that electron's didn't interact with each other but did interact with protons via the Coulomb force. In this universe, exactly solve the Schrodinger equation for a general $\mathrm{N}$ electron atom.

3. Variational Principle II In a previous inquiry, you should have proved that satisfying the time independent Schrodinger equation is equivalent to minimizing something. For most practical cases (for example, computational chemistry), we must approximate and can't quite reach this lowest bound. Typically, we consider only wavefunctions that can be written as a linear combination of a set of basis functions. Given this constraint, express the variational principle as a linear algebra problem (i.e. a matrix equation or eigenvalue problem).

4. Hartree-Fock method If you solved Non-interacting electrons, you should realize that you can take $\mathrm{N}$ single particle states (call them single particle orbitals) and combine them in a special way to create an $\mathrm{N}$ particle state that respects antisymmetry. Suppose that we attempt to use this type of ansatz to represent the state of electrons in a molecule or an atom, where electronelectron interactions are not neglected. Furthermore, as in Variational Principle II, assume that all single particle orbitals are linear combinations of some set of basis functions. Use the variational principle to find a linear algebra problem that represents the "best possible state" given these assumptions.

Concluding notes With the knowledge from chapters 1 and 2, you can make a crack at understanding how quantum computing works. See Quantum Computation and Quantum Information by Isaac Chuang and Michael Nielsen.

\section{CHEMICAL BONDS}

\section{Quick Takes}

1. Lone pairs versus atoms Ammonia and methane are similar molecules, but ammonia is not perfectly tetrahedral as one of its four "ligands" is a pair of electrons, not a bond to another hydrogen. Empirically, we see that the lone 
pair of electrons forces the other bonds to bunch closer together. Compare the validity of this qualitative explanation and the explanation in section $4 \cdot 3$.

2. Hydrogen bond strength Give a back-of-the-envelope estimate for the strength of a hydrogen bond relative to the strength of an actual covalent bond.

\section{Inquiries}

1. Synthesizing sulfur hexafluoride The chemist's mindset is to wonder how every chemical they come across is formed. Propose a reaction to produce $\mathrm{SF}_{6}$ with high efficiency and few toxic reagents and waste products.

2. Aromaticity Warning: this is a fairly advanced problem, so feel free to come back once you've learned a some organic chemistry. Benzene $\mathrm{C}_{6} \mathrm{H}_{6}$ is famous for being aromatic. It has three pairs of $\pi$-electrons delocalized in a "doughnut" around the ring of six carbons instead of the naive three single and three double bonds, giving benzene unique chemical properties. However, $\mathrm{C}_{4} \mathrm{H}_{4}$, $\mathrm{C}_{5} \mathrm{H}_{5}, \mathrm{C}_{7} \mathrm{H}_{7}$, and $\mathrm{C}_{8} \mathrm{H}_{8}$ must gain/lose 1 to 2 electrons to achieve similar properties. Using a simplified version of Hartree-Fock with Coulomb and exchange interactions neglected, mathematically explain this.

\section{MOLECULAR SHAPE AND STRUCTURE}

\section{Quick Takes}

1. Equatorial versus axial See Figures 4.4, 4.5, and 4.6. Can you prove more mathematically why ligands are removed from the positions drawn and not the others?

2. Polarizability of geometric isomers The textbook talks about cis-dichloroethylene being polar and trans-dichloroethylene being non-polar due to symmetry considerations. Which of these two molecules is more polarizable (meaning which molecule's induced dipole moment is larger for a given electric field)?

3. Single-particle approximation Section 4.5 presents a very simplified way of thinking about energy levels in a molecule, where each orbital has an energy and promoting an electron from a lower to a higher energy orbital increases the energy of the system by the difference in energy between the two orbitals. This is known as the single-particle approximation where the single-particle orbitals are the eigenfunctions of the Hamiltonian. In reality, promoting an electron to a higher orbital results in higher-order effects that change the overall system energy not simply by taking the difference between the two orbitals. What are these higher-order effects? Calculating them, a major goal of the field of many body physics, is hard in practice. See Modern Quantum Chemistry by Szabo and Ostlund for more.

4. Combining atomic orbitals Section 4.5 gives equations for the four hybrid orbitals as linear combinations of the atomic orbitals. How were these signs chosen? After all, you can add or subtract each of the four constituent atomic orbitals, resulting in 8 combinations, but there are only 4 . What is special about the four used? When is it not an appropriate approximation to combine the orbitals linearly, as opposed to multiplying them or using higher order products, like squares of the wavefunctions?

5. Hybridizing with d-orbitals To create 5 or 6 hybrid orbitals, we need $1 \mathrm{~s}, 3$ $p$, and either 1 or $2 \mathrm{~d}$ orbitals, respectively. Why not use more $\mathrm{d}$ orbitals and fewer $s$ and/or $p$ orbitals? Is there ever a situation in which using more $d$ orbitals would be better? 
6. Bonding with lanthanides What would the geometry of a molecule comprised of an atom from the lanthanide block surrounded by singly bonded atoms (e.g. $\mathrm{H}$ or $\mathrm{F}$ ) be? What if we threw in some $\mathrm{O}$ to make double bonds or $\mathrm{N}$ to make triple bonds? This molecule probably isn't stable. How could you increase the likelihood of forming it?

7. Sharpening peaks Peaks in IR spectra are typically quite broad. How could you resolve the peaks. i.e. make them sharper?

8. Electronegativity of ions Estimate the electronegativity of an ion, e.g. $\mathrm{O}^{-}$.

9. Forming bands As you know from solid state, as you bond together many, many atoms, you get many, many molecular orbitals which turn into electronic bands. Molecular orbitals, however, are still separated by a discrete amount of energy. Why can we just assume that bands are a continuum of energy states?

10. Surrounding atoms There are multiple geometrical configurations corresponding to 6 ligands surrounding an atom like $S$ in addition to the octahedral configuration. What are these different ways? When would one way be preferred over the other? Propose the most stable geometrical configuration of 8 ligands surrounding an atom.

11. Symmetry breaking Each of $\mathrm{SF}_{6}$ 's 6 bonds are identical. But in some molecular complexes, the symmetry of these bonds can be broken. Why would this occur? Can you think of a model system in which it would occur? Hint: it is related to the occupation of the complexes' orbitals. This is a tough one to figure out on your own, so look up the Jahn-Teller effect if you're stuck.

12. Infinite sulfur and oxygen Assume we have $\mathrm{S}$ bonded to $6 \mathrm{O}$. Each $\mathrm{O}$ can clearly bond to another atom, perhaps $\mathrm{S}$ again, creating an infinitely sized molecule (a polymer) if we kept going. What prevents this from happening?

13. Picking orbitals For a molecule like $\mathrm{SF}_{6}, \mathrm{~S}$ has $\mathrm{sp}^{3} \mathrm{~d}^{2}$ orbitals. Which $\mathrm{d}$ orbitals are hybridized, and which are left alone?

\section{Inquiries}

1. Cis versus trans, spectroscopy Using spectroscopy, how would you tell the difference between cis- and trans-dichloroethylene?

2. $2 \mathrm{D}$ Chemistry Assume we live in a $2 \mathrm{D}$ universe. What would the hydrogenic atomic orbitals look like? What kind of bonding / hybridization would be possible? How would the periodic table change? Is thinking about $2 \mathrm{D}$ chemistry important for understanding chemistry of $2 \mathrm{D}$ materials like graphene?

3. Learning from spectra In these chapters, you learned about IR and UV-vis spectroscopy. There are also x-rays and many other regions of the EM spectrum that can be used to probe molecules to learn things about them. Given a molecule (say cis-dichloroethylene), what can you learn about it from each of these spectra?

4. VSEPR Theory Shapes There is no easy way to model the effective potential between "ligands" on a central molecule. However, even through molecules have ugly potential energy surfaces, VSEPR theory predicts neat, geometrical shapes (i.e. tetrahedrons, trigonal bipyramids, etc.). Using symmetry groups (for example, all five chlorines on $\mathrm{PCl}_{5}$ are identical), can you "bootstrap" these shapes? 
Concluding notes The end of Chapter 4 features a section on drugs from natural products or by rational design, and it talks about the difficulty of synthesizing some of these complex molecules. This challenge is what pulled so many curious minds into chemistry for many decades. Synthetic chemistry in this traditional sense is diminishing in popularity, however, as computers start to design reactions better, and robotic synthesists improve in dexterity and flexibility. Check out the companies ChemAxon and Emerald Cloud Lab. The latter isn't explicitly focused on synthetic chemistry, but it's not hard to imagine the same concept applied to research in pharmaceutical companies like Genetech and Pfizer. This phenomenon speaks to the importance of interdisciplinary work-nowadays, a pure chemist is limited compared to the one who can use tools from a variety of fields.

\section{GASES}

\section{Quick Takes}

1. Higher dimensional universe In a universe with more spatial dimensions, what would the ideal gas law look like?

\section{Inquiries}

1. Van Der Waals Using statistical mechanics, derive the Van Der Waals equation using the cumulant expansion. You can use any ensemble you want, but NVT is the easiest. For the molecule-molecule potential, use an inverse sixth power for $r>r_{m}$ and infinity for $r \leqslant r_{m}$. Using your derivation, explain why the Van Der Waals equation breaks down where it does.

2. Dipole-dipole interaction You should know from electromagnetism that the potential energy between two dipoles scales with the inverse distance cubed. However, the effective potential between two molecules with permanent dipoles will scale differently because molecules at typical temperatures are rotating rapidly. Prove with statistical mechanics that the effective potential scales with inverse distance to the sixth power.

3. London dispersion force Using time independent perturbation theory, prove that two hydrogen atoms at long range will feel an attractive effective potential that scales with inverse distance to the sixth power.

\section{LIQUIDS AND SOLIDS}

\section{Quick Takes}

1. Allotropes Look up the structures of diamond and graphite. Both are pure carbon, yet we see them both under standard conditions. How is this possible? Which do you expect to be more stable under standard conditions? Why is graphite black, but diamond is transparent? How do you expect each to absorb infrared or UV radiation relative to the other?

2. Dissociation curves Figure 6.1 shows the potential energy vs. intermolecular separation $r$ for two atoms, each presumably with some electrons and a nucleus forming a bond. Notably, it's asymmetric. Do you expect this curve to be more or less asymmetric for atoms with higher $Z$ ? This asymmetry curve is often fitted to the Lennard-Jones curve, which sums a positive term that scales with $1 / r^{12}$ and a negative term that scales with $1 / r^{6}$. What is the physical motivation for choosing these exponents (12 and 6), if any? Can you think of a computational reason that these exponents were chosen? Are there more physically motivated choices for these exponents? 
3. Intermolecular interactions Check out Table 6.1. Why is it that dipole-dipole, dipole-induced-dipole, and dispersion (induced-dipole-induced-dipole) have about the same interaction energy?

4. Solvation shells How many waters do you expect a metal ion (e.g. $\mathrm{Ni}^{+2}$, $\left.\mathrm{Cu}^{2+}, \mathrm{Fe}^{2+}, \mathrm{Fe}^{3+}\right)$ to be able to fit in its first hydration shell? Would this depend on, for instance, the metal ion's oxidation level or unoccupied orbitals? How about in its second shell? Third?

5. Scaling laws Figure 6.3 shows how interaction energy scales with distance for different interaction types. Check out The Theory of Intermolecular Forces by Anthony Stone. Chapters 1-4 will build on your EM background, and chapter 5 will give you an idea of how these calculations are done practically. Everything beyond that perhaps is a bit too technical and detailed unless you plan to enter this research field. Can you think of interaction types that scale even more drastically than $1 / r^{6}$ ? Think of an experiment to measure the interaction energy as a function of distance to figure out what interaction type you're probing.

6. Hydrated salts Chapter 6 talks about hydrated salts, and you learn about crystal structures in chapter 7 . Where do you think these water molecules go in the salt crystal structure? Are they dispersed randomly or with order?

7. London dispersion forces Section 6.4 states that because a "large molecule with many electrons is typically more polarizable than a small molecule with only a few electrons, a large molecule is likely to have stronger London interactions than a smaller one." Can you think of a counterexample?

8. Boiling points Do you expect it to be more difficult to calculate from first principles (i.e. using physics and not fitting a curve) the boiling point of a liquid held together with dipole-dipole interactions or van der Waals interactions?

9. Zig zags Why does $(\mathrm{HF})_{\mathfrak{n}}$ form zig zags as opposed to a straight line?

10. Solids as cold liquids A phase transition occurs when there's a discontinuity in some property (such as specific heat in liquid-to-solid transitions) between two phases. If a liquid is simply a jostling, disordered soup of molecules where we can gradually increase long-range order by lowering temperature, why is there a phase transition? Said another way, why can't we just call a solid a cold liquid instead of an entirely new phase?

11. Piezoelectrics In AFM or STM, a piezoelectric actuator is used to precisely control the location of the tip down to the nanometer or even angstrom. Why do we use piezoelectrics as opposed to shape memory materials or other transducing materials (meaning they convert perturbation in some form to another, like electric potential to mechanical in piezoelectrics)? Can you try to come up with an explanation for how piezoelectrics work? What would the crystal structure of a material have to look like to be used as a piezoelectric?

12. Melting points of solids Why do network solids melt at higher temperature compared to ionic solids? How can you predict whether a pure metal will have a higher or lower melting temperature compared to a different pure metal?

13. Brittle materials Why are ceramics brittle, but metals are not?

14. Measuring unit cells Self-test $6.5 \mathrm{~A}$ and $6.5 \mathrm{~B}$ give you the atomic radius of various metals, along with the density, to determine the unit cell. Where do you think they got these atomic radius values? 
15. Radius ratio rules Derive the radius ratio rules for coordination numbers. They pop up all the time, and understanding the derivation is crucial for developing physical intuition of alloys and crystal structures.

16. Graphite's color Why is graphite black but diamond transparent (to visible light)?

17. Liquid crystals Why do some liquid crystals adopt the nematic vs. smectic vs. cholesteric phases?

18. Ionic liquids Why do you think ionic liquids use relatively small anions and large cations rather than vice versa?

\section{Inquiries}

1. Mechanochemistry Imagine you loop a "spaghetti" that is a long, hydrocarbon molecule around another "spaghetti" and pull. How much force would it take to break the "spaghetti"? This is not just a thought experiment! Mechanochemistry is a real, thriving field. Reactions are often done in sonicators, which are devices that vibrate the water in a bath. They're typically used to prevent or break down aggregates in solutions when doing chemical synthesis or preparing cell buffer for tissue culture, but an adventurous chemist found that when the microbubbles that these sonicators create pop, enormous pressures are induced locally that can change the course of chemical reactions.

2. Life without hydrogen Could life exist without hydrogen? Questions like these are asked at, for instance, the NASA Astrobiology Institute. They do some awesome stuff there. Check it out if you're interested, especially in research internships for high school students.

3. Femtosecond measurements Propose an experiment to track the instantaneous dipole moment of a nonpolar molecule with a resolution of $10^{-16} \mathrm{~s}$. Hint: feel free to look up some specs of lasers, single photon sources, and single photon detectors.

\section{INORGANIC MATERIALS}

\section{Quick Takes}

1. Metallic mirrors As Section 7.1 discusses, metals can reflect light because electrons can oscillate with the light and re-emit the light, and evidently this is how metallic mirrors work. Mirrors can have reflection efficiencies up to $99.999 . . \%$. This seems impossible under the naive assumption that the light is re-emitted uniformly in a sphere from the oscillating electrons. How does the light that gets emitted into the bulk of the metal find its way back out? What happens when the frequency of the incoming light is higher than the maximum frequency at which the electrons can oscillate (the plasma frequency)?

2. Opacity Estimate the minimum thickness of, say, silver needed to be completely opaque. Feel free to look up any numbers except the absorption coefficient.

3. Malleability and ductility Books often distinguish between malleability and ductility, and naively one would expect a material that is malleable to be ductile. When would this not be the case?

4. Eutecticity Why would some alloys be eutectic and others not? 
5. hBN vs. graphene Look up a picture of graphene, which is a single layer of graphite. Now imagine replacing half the atoms with boron atoms and the other half with nitrogen. You get hexagonal boronitride, which is a wellstudied 2D material. How do you expect its electrical and thermal conductivity to change relative to graphene? What color do you think it is compared to graphene? What about its stiffness?

6. Glassy metals Say we wanted to cool some liquid metal as fast as possible so it doesn't have time to crystallize, resulting in a glassy metal which has many interesting properties. How would you do it?

7. Qubit transmission Say we want to send a qubit of information in the form of a photon, where $|0\rangle$ corresponds to horizontal polarization of light and $|1\rangle$ corresponds to vertical polarization, to a quantum computer $1000 \mathrm{~km}$ away. Why couldn't you simply send it down an optical fiber? Is there an ideal photon frequency to use? This is why quantum networks require quantum repeaters! See https://qt.eu/understand/underlying-

principles/quantum-repeaters/

8. Maglev trains Why do maglev trains require tracks made of superconductors as opposed to any generic conductor?

9. Spin flipping Why does flipping a spin in phosphorescence take so much longer than simple orbital transitions in fluorescence?

10. Antiferromagnetism Antiferromagnets have no magnetic moment because the spins are anti-aligned, but many other materials, such as glass, also don't have a magnetic moment. How could you tell the difference between an antiferromagnet and these other materials?

11. Usefulness of nanomaterials There's been lot of hype about nanomaterials, such as carbon nanotubes and nanoparticles, for decades, but their real-world applications have historically been quite limited. Can you think of a few reasons why?

\section{Inquiries}

1. Metallury Without looking up gold alloys, design your own gold alloy that's harder than gold (which is notoriously ductile) using atomic radii info and your knowledge of crystal structures. How do you expect the color to change? After you design it, see if it exists.

2. Graphene synthesis How would you synthesize graphene from the bottomup (using individual carbon atoms) rather than top-down (starting with, say, graphite)?

3. Solid electrolyte A solid electrolyte conducts ions, not electrons. How do you expect the charge conductivity of a solid electrolyte to compare to a metal? Describe a structure where it would be possible to conduct ions but not electrons.

Concluding notes One of the scientists who won the Nobel Prize for creating graphene by peeling it off graphite with Scotch tape is the only scientist to have also won the Ig Nobel Prize for floating a live frog with magnets. He says he stumbled upon both ideas during Friday Night Experiments, where his group would just try whatever they found interesting. Just a fun story that reminds us that scientists can be nothing but curious kids who treat nature as a playground. 


\section{THERMODYNAMICS: THE FIRST LAW}

\section{Quick Takes}

1. Thermodynamic scaling Expanding infinitely fast is not thermodynamically efficient at all, and expanding infinitely slowly is maximally thermodynamically efficient. What happens in between these expansion rate regimes? In other words, how does efficiency scale with expansion rate?

2. Conductivity of phases Is it possible to have a gas that conducts heat better in one direction over another gas? How about a liquid? A solid?

3. Heat capacity of phases What would have a higher heat capacity: crystalline or amorphous (basically glass) silicon oxide? Can you think of a reason for the difference? Hint: Think about the vibrational (phononic) band structures. Will this trend flip upon increasing or decreasing the temperature?

4. State functions What are some examples of properties or objects that are state functions in quantum mechanics? In E\&M?

5. Thermocouples Thermocouples are thin probes that experimentalists use to locally measure the temperature. Using the knowledge that they rely on the Seebeck effect, figure out how they work.

6. Better than water The end of Section 8.12 says, "The high heat capacity of liquid water is due largely to the extensive hydrogen-bonding network that survives in the liquid. The molecules are linked to one another by loose intermolecular hydrogen bonds, and the vibrations of these "bonds" can take up energy more readily than can the stiff chemical bonds between atoms." Why is this the case? Given the approximate energies of hydrogen bonds vs. other intermolecular bonds and of different types of hydrogen bonds, name a substance in liquid form that you expect to have even higher heat capacity than liquid water.

7. Standards for heat of formation Setting the zero of enthalpy of formation to that of an element's most stable form might seem arbitrary. Does it matter whether we pin the zero of enthalpy of formation to an element's most stable form vs. some other form?

8. Bond formation If bond-forming is always exothermic, why can't some molecules be formed?

9. Hydrogen-fueled cars For a while, people thought hydrogen-fueled cars would overtake their gasoline- and electricity-powered counterparts. Why do you think this didn't end up happening? If you're curious to learn more, check out Physics for Future Scientists by Richard Muller who talks extensively about the failings of hydrogen-fueled cars.

\section{Inquiries}

1. Thermal isolation Let's say we wanted to study the thermodynamic properties of some liquid. Sketch out a design for a chamber to isolate this system as much as possible from the environment.

2. Thermodynamic baths Whatever system we study, everything else in the universe is deemed the surroundings, or the environment, or the bath in physics communities. Are all baths the same? How can the properties of one bath differ from another? 
3. "Adiabatic" "Adiabatic" is a confusing term because it seemingly applies to many rates at both the slow and fast end. On the fast end, there are examples in computational chemistry and thermodynamics. In computational chemistry, the Born-Oppenheimer approximation is often described as an adiabatic approximation, where electrons move so much faster than nuclei that you approximate the nuclei as being frozen. In thermodynamics, adiabatic expansions occur when the rate of expansion is so fast that heat doesn't measurably escape. On the slow end, we've got the adiabatic approximation in quantum mechanics, where you assume that the system evolves so slowly that the system does not hop energy levels, allowing you to make drastic simplifications in the time-dependent Schrodinger equation. Given these seeming "contradictions," concisely and precisely define what "adiabatic" means.

4. Molecular heat capacity A linear molecule has 2 rotational modes, which contribute $2\left(\frac{1}{2}\right) k_{\mathrm{B}} T$ to the heat capacity through the equipartition theorem, while a nonlinear molecule has 3 rotational modes. What about a molecule that's just slightly non-linear? How could you force a linear molecule to be non-linear? What applications can you imagine for controlling a molecule's heat capacity by controlling its geometry?

5. Thermodynamics of information There is a one-to-one connection between thermodynamics and information theory. A well-known thought problem demonstrating this connection is Maxwell's demon. It goes something like this: Imagine two connected boxes filled with gas. The box on the left is filled with cold gas and the one on the right is filled with hot gas. Gas particles can travel between the two boxes via a small window controlled by "Maxwell's demon". Maxwell's demon has full knowledge of every incoming particle's momentum and position, so it can decide to open the window when a relatively fast particle in the cold gas approaches, releasing the fast particle in the hot gas and thereby increasing making the cold gas colder and the hot gas hotter. Overall, entropy has decreased! This obviously breaks the second law of thermodynamics and is quite the paradox. How do we resolve this? Partly adapting from Wikipedia, it turns out that you have to include the entropy change of collecting information: For Maxwell's demon to know how fast the gas particle is moving and where it is, it must do some sort of measurement, and for a while it was argued that this measurement would generate more entropy than letting the fast particle through would take away. Then it was discovered that that's not necessarily the case, so people had to find something new to point at. That's when they released that the demon would have to store the information and at some point would run out of space and delete it, which increases entropy and recovers the second law of thermodynamics. There have been a few more back-and-forths, so resolving the paradox is an ongoing argument. Here is a thought experiment that complicates matters: Instead of atomic-sized gas particles, what if we had macroscopic balls flying around in these two boxes and Maxwell's demon let one of these macroscopic balls out from the cold box to the hot box? Sure, there'd be entropy gain from deleting the storage of the velocity and position of the center of mass of a macroscopic ball, but the change in entropy from the cold to the hot box would be enormous since we'd have many, many atoms in this macroscopic ball being shifted over. The field of information theory was founded by Claude Shannon for his Master's thesis. You can read more about him and other legends of Bell Labs in The Idea Factory.

\section{THERMODYNAMICS: THE SECOND AND THIRD LAWS}

\section{Quick Takes}


1. Total entropy on earth Very, very roughly, how much entropy is there on earth? Very roughly, what is the rate of entropy change from incoming sunlight? Scientists and philosophers love attributing entropy as the cause of many things, including the existence of life! See the overview on Wikipedia and the popular news article on Quanta Magazine titled "A New Thermodynamics Theory of the Origin of Life".

2. Entropy over a phase transition Plot the entropy of something that's initially liquid and boils off. Put entropy on the $\mathrm{y}$ axis and reaction progress on the $\mathrm{x}$ axis.

3. Entropy of enthanol versus water Why does ethanol have a higher entropy of vaporization at the boiling point than water?

4. Lattice model of entropy In the lattice model of entropy, some number of atoms are assumed to occupy or not occupy discrete lattice points. The entropy can be calculated using the number of equivalent microstates possible. See Figure 9.7 for an illustration. Confusingly, if we increase the density of lattice points for some volume of a crystal but kept the number of atoms constant, then we'd calculate more possible microstates and thus higher entropy. But entropy is a state function and shouldn't depend on how we define our grid of lattice points. How do you reconcile this seeming contradiction? This question is especially relevant when calculating the entropy of, say, a gas or lattice where the atoms don't simply occupy crystal lattice sites using the lattice model.

5. Entropy of breathing When you (the system) respire, does your entropy increase or decrease?

6. Helmholtz versus Gibbs Gibbs free energy measures the maximum work possible from a closed system at constant temperature and pressure. It's useful in chemistry because most reactions of interest do occur at constant temperature (enforced with a thermostat) and pressure (because the reaction is open to the environment, which is much larger than the reaction bath, so even if a bunch of gas is produced in a reaction, the overall pressure should remain about the same). We could also define the Helmholtz free energy, however, which measures the maximum work possible from a closed system at constant temperature and volume. When would the Helmholtz free energy be useful? How can you calculate the Helmholtz free energy from the Gibbs free energy?

7. Rate of ATP consumption We create ATP, which is responsible for driving mostly everything your body does with energy, from ADP by oxidizing glucose, where the oxygen comes from breathing. Assume a human dies or passes out when he/she runs out of ATP. About how long can a human hold his/her breath? You'll have to Google some numbers.

\section{Inquiries}

1. Quantum thermodynamics Is the third law of thermodynamics compatible with quantum mechanics? This question opens a giant can of worms, so we don't expect too much. It's just to get you thinking before checking out the Wikipedia article on quantum thermodynamics and the seminal paper by Linus Pauling.

2. Photonic fluids Maximum efficiencies of closed processes are often calculated by envisioning a heat engine, where the maximum efficiency is achieved with an engine running the Carnot cycle and is straightforwardly defined in terms of the temperatures of the hot and cold baths. For a closed process involving others forms of energy, say sunlight being absorbed by solar panels which is converted to chemical energy in the form of a battery that then drives an 
LED, determining the maximum efficiency is not as well-defined. Is the efficiency for this process even limited by the Carnot efficiency? How would you calculate it?

3. Atomic computers Atkins states that "at very low temperatures an interference pattern of laser beams can be used to create an 'optical lattice,' a set of spatially symmetric potential energy wells in which atoms can be trapped to form perfect crystals. Apparently, "these arrays could be used to create tiny building blocks for very-low temperature nanoscale computers." Briefly explain how a computer made from this architecture would work.

\section{Quick Takes}

1. Transition state theory We can use Gibbs free energy to calculate which direction a reaction will go, but can it also tell us how fast it will move? For more, look up transition state theory.

2. Electrolyte maintenance Table 10.1 lists the concentration of principal ions found in seawater. Compare that to the concentration of these ions in organisms, such as bacteria, fish from the sea, and humans. What ions do these organisms have to concentrate vs. dilute in their bodies? What are the implications?

3. Volume-dependent vapor pressure Figure 10.1 says that the "vapor pressure is the same however much water is present in the column." This isn't precisely true. How would you calculate the pressure change when you add more water? What happens to the vapor pressure as you decrease the water content to nothing?

4. Equilibrium time Let's say you have some water in a cup and you suddenly seal it. Approximate how long it would take for the vapor pressure to reach equilibrium. This time is dependent on the system geometry-how could you maximize or minimize this time?

5. Vapor pressure of solids Do you expect graphite or diamond to have a higher vapor pressure at room temperature? Will one have a higher vapor pressure than the other for all temperatures, or is it possible for their respective vapor pressures to cross over at some temperature? Explain why or why not.

6. Clausius-Clapeyron for solids Does the Clausius-Clapeyron equation only apply for the vapor pressure of liquids, i.e. can we use it to predict the vapor pressure of solids?

7. Breakdown of Clausius-Clapeyron What are some situations where ClausiusClapeyron equation would break down?

8. Pressure-dependent phase Will a higher density phase always be more preferable at higher pressures?

9. Phase diagrams with different variables The idea of a phase diagram is much broader than plotting temperature and pressure on the axes. What kinds of phases would be affected by electric field? Magnetic field? Irradiation intensity?

10. Hydrated salts and aqueous solutions At what point does a hydrated salt become an aqueous solution of salt? 
11. Solvation energies Estimate the solvation energy of a nonpolar molecule like hexane dissolving a nonpolar liquid, such as octane, and compare it to that of salt dissolving in water.

12. Solubility in an ionic liquid What kind of solute would best dissolve in an ionic liquid?

13. Getting rid of scum "Scum", the precipitate of magnesium and calcium ions in hard water with the fatty carboxylic acids of soaps, can be found on shower drains and shower heads. (Check your drain to get a sense for how hard the water in your area is.) How would you get rid of the scum chemically?

14. Surfactant precipitation Why do surfactants with sulfur atoms in their polar groups not form precipitates with the ions in hard water?

15. Nitrogen solubility In a glass of water sitting in atmospheric conditions (room temperature and $1 \mathrm{~atm}$ of pressure), estimate the ratio of nitrogen molecules (which could only come from atmospheric gas) at the bottom of the glass to the top of the glass.

16. Henry's constant What factors go into Henry's constant for gases, i.e. why would the Henry's constant for gas A be different than gas B? Better yet, loosely derive an expression for Henry's constant.

17. Temperature-dependent gas solubility Why is gas solubility lower at higher temperatures? What shape should the curve be of solubility vs. temperature, e.g. linear, quadratic, etc.? Why would a small number of compounds show mixed behavior?

18. Minimizing solute-solute interactions Estimate how dilute a solution would have to be so that you can ignore solute-solute interactions when measuring the limiting enthalpy of solution. Why not just dissolve a single molecule into a solution to measure this quantity?

19. Units of concentration Molality may be temperature independent, but practically all chemists and biologists will use molarity instead. Why?

20. Freezing point constant What factors go into the freezing point constant $k_{f}$ and van't Hoff factor i, i.e. why would they be different for gas A than gas B? Better yet, loosely derive expressions for them.

21. Isolating pure water Which would be a more energy efficient way to isolate pure water from salt water: squeezing salt water through a semipermeable membrane via reverse osmosis, or boiling salt water and collecting the water vapor?

\section{Inquiries}

1. Inverse salt factory Isolating salt from seawater requires inputting energy, so mixing it back together should release energy. Sketch out a design for a machine that could take advantage of this 'free energy of mixing' to produce work.

2. Quadruple point How could you prove that a quadrupole point on a phase diagram could never exist, i.e. what conditions must be true? This is a tough problem, so feel free to look up the solution after a bit and try to understand it. For modern research on the topic, see "A possible four-phase coexistence in a single-component system" in Nature Communications, an open-access journal. 
3. Measuring gas solubility Let's say you want to measure the solubility of a gas based on the number of tiny bubbles that form as you increase the temperature. How would you design this apparatus, and what kind of experimental challenges do you anticipate?

4. Thermodynamics of azeotropes Say we have a minimum-boiling azeotrope whose boiling point is reduced relative to those of the two, pure constituent solutions. This is naively a troubling concept from an energetic perspective because we know that for a pure solution, the total energy of the liquid (potential energy from the molecules interacting with each other and some kinetic energy as they slowly diffuse around) plus the energy that goes into heating should equal the energy of the boiled liquid (gas molecules zipping around with some kinetic energy), but in an azeotropic liquid, to get the same amount of liquid to boil and up to the same temperature as the boiled liquid from a pure liquid state, we don't need to input as much energy. Reconcile this seeming contradiction.

5. Drug delivery Tissue engineering and drug delivery are thriving subfields of chemistry / materials science / bioengineering. (If you are interested in research in these fields, some places with summer programs for undergradutes include the Wake Forest Institute for Regenerative Medicine and the McGowan Institute for Regenerative Medicine.) Choose a colligative property you've learned about in this chapter, and propose a mechanism for drug delivery by manipulating this particular property.

\section{CHEMICAL EQUILIBRIA}

\section{Quick Takes}

1. Mass action Derive the law of mass action.

2. Activity approximation We assume the activity of a pure solid or liquid is just 1. When would this approximation break down, and how would you correct for it?

3. Non-ideal activities Name gases that you expect to have a higher or lower activity for a given concentration than an ideal gas.

4. Imperfect catalysis A perfect catalyst should not affect the equilibrium constant. (If you haven't answered the thinking point on page 432 yet, then explain why.) Propose a couple ways that a catalyst could be imperfect, and derive expressions for how the rate of the reaction of interest would be impacted. To get a running start, look up Michaelis-Menten kinetics.

5. Pressure and concentration Relate $K_{p}=K$ and $K_{c}$ for a reaction with an arbitrary number of chemical species in liquid or solid state.

6. Limits of Le Chatelier's Le Chatelier's principle is quite general. Mathematically and generally, explain it. Name some examples beyond chemistry that uphold or don't uphold Le Chatelier's principle.

7. Temperature-dependent entropy change In the derivation for the van't Hoff equation, $\Delta S_{r}^{o}$ is assumed to be temperature independent, so the van't Hoff equation only has enthalpy in it. What is an example of a reaction with $\Delta S_{r}^{o}$ that is likely to be very temperature dependent?

\section{Inquiries}

1. Non-equilibria Why are systems not at equilibrium often both more interesting and more difficult to solve than those at equilibrium? 
2. Limits of known equilibrium constahts Estimate the maximum and minimum equilibrium constant one could practically measure. You will have propose or look up methods to measure equilibrium constants.

3. Catalyst criteria Catalysts are typically made of a metal or a metal oxide. How do they accelerate reactions? Google some catalysts for famous reactions (such as the Haber catalyst) and come up with some design criteria for the composition of catalysts.

\section{ACIDS AND BASES}

\section{Quick Takes}

1. Superacids Superacids, such as fluoroantimonic acid and trifluoromethanesulfonic acid, are acids with an acidity greater than that of $100 \%$ pure sulfuric acid. Why are they such strong acids?

2. Water clusters Estimate how many water molecules there would be in the most stable cluster of water molecules with a single hydrogen ion $\left(\mathrm{H}^{+}\right.$vs. $\mathrm{H}_{3} \mathrm{O}^{+}$vs. ...)

3. Time of deprotonation Does the time scale at which an acid is deprotonated depend on its acidity? What about the protonation rate of its conjugate base? What is this time scale (femto- vs. pico- vs. nano- vs. milli-seconds?)

4. Mass-charge balance The classic example demonstrating the need for masscharge balance is, what is the $\mathrm{pH}$ of a solution with $10^{-} 8 \mathrm{M} \mathrm{HCl}$ ? Without mass-charge balance, you'd get a $\mathrm{pH}$ of 8 , which is obviously wrong because we've added acid into the solution. What approximation break down that give us the wrong answer?

5. Acid to base Why do the oxides of elements that lie on the diagonal frontier between the metals and nonmetals gradually shift between acidic and basic character?

6. Temperature-dependent acidity Given the temperature dependence of the autoprotolysis of water, do you expect acids and bases to become weaker or stronger at higher temperature?

7. Autodissociation How would the autodissociation constant of water in gas phase be affected by pressure, volume, temperature, and number density?

8. Voltage between electrodes Estimate the voltage difference between two electrodes separated by distance $\mathrm{d}$ immersed in a solution with $\mathrm{pH}$ of $\mathrm{o}$.

9. Waste management Based on Figure 12.12, the $\mathrm{pH}$ threshold for waste being corrosive depends on whether it is an acid or base (about $5.5 \mathrm{pH}$ units from $\mathrm{pH} 7$ for bases, but only $4 \mathrm{pH}$ units from $\mathrm{pH} 7$ for acids). This observation suggests that whatever is affected by waste, such as biological processes, is more sensitive to base than acid. Why?

10. Measuring heat of solvation Based on Table 12.4, why is it so difficult to measure $\Delta \mathrm{H}_{\text {solv }}(\mathrm{HA})$ for $\mathrm{HCl}, \mathrm{HBr}$, and $\mathrm{HI}$ ? Propose a better method.

11. Strength of polar bonds In Table 12.7, trends for acid types are given. Is it generally true that a more polar bond is weaker? Why or why not?

12. Acid comparison Would you expect $\mathrm{H}_{2} \mathrm{SO}_{4}$ to be a stronger acid than $\mathrm{H}_{2} \mathrm{SO}_{4} \mathrm{~F}_{4}$ ? 
13. Polyprotic acids The $\mathrm{pK}_{\mathrm{a}}$ increases by about $3-5$ for each successive deprotonation in a polyprotic acid. Justify this empirical order of magnitude observation.

14. $\mathbf{p H}$ of polyprotic acids Derive equation 14. How would you generalize it to an acid that can donate three protons, such as phosphoric acid?

15. Limits of mass-charge balance How weak does an acid have to be for it to be necessary to take into mass-charge balance to achieve, say, $5 \%$ error?

\section{Inquiries}

1. Superbases Design a couple molecules that you think could be superbases. Check your design against literature.

2. Measuring acidity Measuring the strength of an acid in the traditional fashion by measuring its degree of dissociation in water doesn't work beyond a certain level of acidity. What is this threshold? Propose a method to measure the strengths of acids beyond this threshold.

3. Battery chemistry The concept of acidity and basicity corresponding to the propensity of a molecule to give up or accept a hydrogen atom can be generalized to other elements. Studying the "acidity" and "basicity" of lithiumcontaining compounds, for instance, would be helpful in understanding solidstate lithium-ion batteries, which power all sorts of devices from Teslas to iPhones. What sorts of ideas/concepts/physical phenomena can we transport directly from traditional acid/base chemistry, and what differs? Hint: consider the differences between a liquid solution and a solid-state compound.

Concluding notes Acid and base chemistry forms a bulk of the experiments you'll typically do at the high school and early undergraduate level, so here are some notes on good practice in the lab. Firstly, due to sodium hydroxide's natural tendency to absorb both carbon dioxide and water from the air, practically speaking you need to be very careful with how you store and handle sodium hydroxide, or your mass measurements will be off. You need to be even more careful with sodium hydroxide solutions, however. Because these solutions are basic, they readily absorb carbon dioxide from the air (explain why), and over time, you'll find that your actual $\mathrm{pH}$ can differ from your desired one by up to $10 \%$ of your original sodium hydroxide concentration! This is why in many labs, sodium hydroxide solutions are made day of for experiments. Another important tip is to "Always remember to acid to wata [water] because you oughta!" is what one of the authors' high school chemistry teacher would always tell us in a grotesque caricature of a Boston accent. He has a point though, as many a chemist who has neglected this advice has been burned by splashing, boiling acid. Explain why acid should be added to water as opposed to the other way around.

\section{AQUEOUS EQUILIBRIA}

\section{Quick Takes}

1. Buffers of weak acids and bases If a "mixture of a weak acid and its salt will act as a buffer at $\mathrm{pH}<7$ (the acidic side of neutrality). . . [and a] mixture of a weak base and its salt will act as a buffer at $\mathrm{pH}>7$ (the basic side of neutrality)", what if we have a mixture of the conjugate base of a weak acid and its salt? Would it be acidic or basic? Why?

2. Low concentrations of acid and base Calculate the $\mathrm{pH}$ of a solution with $10^{-8} \mathrm{M} \mathrm{HCl}$ and $10^{-8} \mathrm{M} \mathrm{NaOH}$. Feel free to use Mathematica or some other software if you'd like. 
3. Precise calculations The text states that "[b]ecause our calculations interpret activities as molarities, not effective molarities, they ignore ion-ion interactions; so the values calculated are only approximate but are a reasonably good guide (and a good starting point for more elaborate calculations)." How would you make your calculation an order of magnitude more accurate?

4. Salt clusters Apparently, "a saturated solution of $\mathrm{PbI}_{2}$, for instance, contains substantial concentrations of $\mathrm{Pb}^{2+} \mathrm{I}^{-}$and $\mathrm{Pb}_{2+}\left(\mathrm{I}^{-}\right)_{2}$ ion clusters." Justify why this might occur. How would you determine this to be the case experimentally?

5. Measuring solubility Approximately what $\mathrm{K}_{\mathrm{sp}}$ corresponds to the boundary between "basically not soluble at all" (beyond what could be measured easily) and "sort of soluble."

6. Estimating solubiity Predicting solubility given $K_{\mathrm{sp}}$ isn't much of a challenge computationally (but, of course, you should still learn how to do it manually!) Computing $\mathrm{K}_{\mathrm{sp}}$ itself, however, is difficult. Design the equivalent of a BornHaber cycle for solubility.

7. Isotopic solubility How do you expect $\mathrm{K}_{\mathrm{sp}}$ to differ for some chemical compound if all of the atoms of one of the elements were replaced with their isotopes?

8. Cyanide Check out Table 13.5. Note that formation constants with cyanide are extremely high. Why? You might not fully understand why until you get into organic chemistry or molecular orbital theory, but it's good to start thinking about it now.

9. Elemental analysis In practice, hardly anyone does qualitative analysis by taking advantage of solubility constants. It's simply too noisy and environmentdependent. Suggest some other ways to identify what a compound is made of.

\section{Inquiries}

1. Automatic titration In practice, generating a titration curve by slowly adding acid/base via burette to a target solution of base/acid is incredibly tedious; you have to add progressively less titrant to make sure you don't overshoot the endpoint. Getting the end point is annoying also because as you get very close to it, the solution takes longer to equilibrate upon addition of titrant acid/base; you have to stir the solution up to minutes sometimes. It's also difficult to read the end point because the $\mathrm{pH}$ vs. volume of titrant added changes slope so rapidly. How would you improve this process? (Some ideas I'm hinting at here are plotting something other function or designing some sort of PID algorithm.)

2. Titration with weak acids and bases "Weak acids are not normally titrated with weak bases, because the stoichiometric point is too difficult to locate." Would any of your ideas in response to the previous question alleviate this issue? If not, think of one that would.

3. Indicator optimization Explain generally why colorimetric indicators change color upon addition/removal of a hydrogen ion. Note that most of the indicators in Table 13.3 change to a color that is lower in wavelength going from acid to base. Why is this? Ideally, you're looking for an indicator whose two colors on either side of the endpoint are starkly different from each other (very different wavelengths, like alizarin), but indicators like these are rare in practice. Why? How could you shrink the $\mathrm{pH}$ range in which the color of an indicator changed? 


\section{ELECTROCHEMISTRY}

\section{Quick Takes}

1. Rates of electrochemical reactions Electrochemical reactions, e.g. oxidation of permanganate by oxalic acid, often have complex and large stoichiometry coefficients. If "standard" reactions, e.g. different species of gases reacting with each other, had such complex and large stoichiometry coefficients, the reaction probably wouldn't move very fast, if at all. Why is this? Why can electrochemical reactions happen despite the complex and large stoichiometry coefficients? Engineering kinetics in electrochemistry is often a dark art, so it continues to be an area of active interest.

2. Battery design What battery chemistries would give a) the highest voltage, $b$ ) the highest current per volume, and c) highest energy density?

3. Inertness What value of what property does a conductor have to be for it to be "chemically" inert enough to be used for the hydrogen gas half-cell?

4. Potato battery A popular science demonstration for kids is lighting a bulb with potatoes as the energy source. What voltage do you expect to get from a single potato?

5. Irreversible battery operation Estimate the potential of a working cell operating irreversibly.

6. Salt bridge kinetics In a salt bridge, what are the implications of when the migratory speeds of the cations and anions are drastically different from each other?

7. Standard potentials in non-aqueous solutions Using standard potentials is a nice way of calculating equilibrium constants, but how would you adapt this procedure for non-aqueous solutions, e.g. gases, solutes in solvents that aren't water, etc.?

8. Generalized electrochemical sensor What chemistry would you use to make a Cl-sensitive electrode, i.e. to measure $\mathrm{pCl}$ ?

9. Standard potential of an alloy In the Downs process, steel cathodes are used to reduce sodium ions to sodium, while a pure iron electrode would not work for this application. How would you calculate the standard potential of an alloy?

10. Non-rechargeable batteries Looking at the chemistries of primary cells in Table 14.2, why can't these batteries be recharged, and why can the chemistries of the secondary cells be recharged?

11. Ion conductors A crucial component of solid state batteries is an ion-conducting, but not electron-conducting, interface. How do they work?

12. Nuclear battery Is it possible to create a nuclear battery, where instead of using electrons to transfer energy, the nuclei transfer subatomic particles and radiation?

\section{Inquiries}

1. Long-lived free electrons In Section 14.1, the text states that an "oxidation half-reaction is a conceptual way of reporting an oxidation: the electrons are never actually free." This isn't always true, in fact. What would be the use of a long-lived free electron? How would you engineer the system to observe it? See the "solvated electron" article in Wikipedia. 
2. Noisy electrochemical experiments Electrochemistry is one of the most precise and quantitative subfields of chemistry, especially for measuring reaction rates and thermodynamic properties (why?), as the text describes. This also means, however, that electrochemical experiments have to be controlled very precisely. What are some potential general sources of an error in an electrochemical experiment, and how can you protect against them?

3. Coin battery Figure out a way to make a battery from coins. If you have the resources, try it out yourself!

4. Lithium dendrites Batteries are simple in the ideal case, but in reality, they are messy, sensitive devices. For instance, a major limitation in the development of long-lasting lithium-ion batteries was the formation of dendrites of lithium metal through electrodes, shorting the battery and generating media frenzies when batteries would light on fire in airports. Look up some common lithiumion battery designs and chemistries, and suggest some improvements.

5. Minimizing overpotential Suggest some ways of minimizing overpotential.

\section{CHEMICAL KINETICS}

\section{Quick Takes}

1. Reaction timescales Rationalize why the time scale of a chemical reaction, e.g. molecule A dissociating or molecules $\mathrm{B}$ and $\mathrm{C}$ reacting to form $\mathrm{D}$, can be as fast as a femtosecond.

2. Multiparticle collisions Reaction mechanisms involving 3 or more particles interacting at the same time are typically disregarded because the convention is that getting three particles to collide is statistically unlikely. Estimate the relative probability of a two-particle collision vs one with three particles. Can you generalize to $n$ particles?

3. Physical interpretation of reaction orders Rationalize why does the expression for the half-life of second-order reactions includes the concentration of the reactant, but the half-life of first-order reactions does not.

4. Zeroth order reactions What are some examples of zeroth order reactions? Is it possible to catalyze a zeroth order reaction?

5. Simplifying reactions For the equation for the net rate of formation of $\mathrm{N}_{2} \mathrm{O}_{2}$ at the bottom of page 634, are there any approximations you can make right when the reaction starts (not when it's been going on for a while and is at steady-state)? What about in between these two time regimes? Numerically plot the reactant and production concentrations vs. time using both your approximations and the actual equation.

6. Reactive hydrogen atoms Are two $\mathrm{H}$ atoms with a single electron each more reactive toward each others, or an $\mathrm{H}$ atom with a + charge and an $\mathrm{H}$ atom with a - charge?

7. Arrhenius equation in solids The text explicitly states that the Arrhenius equation applies to reactions of gases and in solution. What about of solids?

8. Temperature-dependent Arrhenius coefficient The text states that the A factor in the Arrhenius equation is independent of temperature, but in fact, it is not. Learn about the derivation of the $A$ factor and explain why it's often justified in practice to say that it is independent of temperature. A related question: It's typically difficult to predict reaction rates any better than within 
a single order of magnitude. Why? Because biological reactions generally follow the Arrhenius equation, you'll find that, among other interesting implications, animals that have lower body temperatures typically live longer and bugs (which are tiny and thus their body temperatures match the environment) are physically unable to be active below some temperature!

9. Bosonic and fermionic reactions How would the temperature dependence of reactions of bosonic particles differ from that of fermionic particles based on collision theory?

10. Multidimensional potential energy surfaces How many dimensions would the potential energy surface of the reaction between a hydrogen molecule and a bromine atom have?

11. Quantum reaction kinetics Is transition rate theory compatible with quantum mechanics?

12. Catalyst poison Why does lead serve as such a potent poison for many heterogeneous catalysts? Estimate what other heavy metals might serve as potent poisons.

13. Enzyme mechanisms Explain how the induced-fit mechanism for enzymesubstrate interactions suggests that there is an optimal temperature for enzyme operation, while the antiquated lock-and-key perspective does not.

\section{Inquiries}

1. First principles calculations of rate constants Solving kinetics problems is as easy as crunching equations in Mathematica (or as hard as solving them analytically). The really challenging part is coming up with the rate constants from first principles. Starting from the theory of chemical kinetics of your choice (transition state theory, Marcus theory, Arrhenius theory, etc.), outline what calculations you would do to get rate constants.

2. Tracking reactions Go into a bit more detail on how tracking reactions on the attosecond time scale works without looking it up, and then look it up to validate your guess. Some details to think about: how many molecules can be monitored at a time, what sample sizes are necessary to get trustworthy results, how to produce attosecond pulses, what aspect of the molecule does the laser pulse actually measure (Box 15.1 discusses optical absorption, but are there other properties that could be measured? Is this absorption driven by electronic excitations, nuclear excitations, IR, Raman, etc.?), how this aspect is measured.

3. Mixing reagents Design a system that mixes reagents as quickly as possible (since just letting the reagents diffuse into each other might be too slow) so that the start time of reactions can be determined with high precision.

4. Limits of tracking reactions Estimate the fastest and slowest measurable reaction rates.

5. Automatic determination of reaction mechanisms Coming up with reaction mechanisms to match observed rate laws is tedious work. Come up with an algorithm that automatically does this.

6. Capturing reaction intermediates A powerful way to deduce reaction mechanisms is to isolate the intermediate (say, a bare atom or a particle in a highly charged or geometrically unfavorable state), which is difficult because its lifetime is so short. Design some ways to capture and identify reaction intermediates. 
7. Forcing multiparticle collisions One way to engineer novel reaction mechanisms is to use three- to n-particle collisions that aren't often experimentally observed, which you should prove to yourself in the question above. Design a way to force three- to n-particle collisions.

8. Messy kinetics experiments Say we do a kinetics experiment and find messy orders, e.g. the exponent of the concentration isn't the rate equation isn't a convenient $1,2,1 / 2,2 / 3$, etc. Perhaps the reaction order seems to be different for different concentrations or temperatures. What are some explanations for this?

9. Direct measurement of reaction rate Instead of measuring concentration vs. time and plotting, for instance, the natural logarithm of concentration vs. time to see if it's 1st order, how can we measure the reaction rate directly, i.e. what's output by your experimental detector is the reaction rate, not the concentration?

10. Steric factors Explain the origin of the "cone of successful attack." Similarly, how would you roughly calculate the empirical steric factor P?

\section{THE ELEMENTS: THE MAIN-GROUP ELEMENTS}

\section{Quick Takes}

1. $\mathbf{d}$ orbitals "Elements in Period 3 and higher periods can have higher valences than the octet rule suggests" because "[t]hese elements have access to empty $\mathrm{d}$-orbitals and can use them to expand their valence shells past the usual octet of electrons." Why is expanding to empty d-orbitals more favorable than expanding to s- or p-orbitals, thus allowing these elements to break the octet rule?

2. Orbital overlap Estimate the difference in degree of overlap between p-orbitals in $\mathrm{N}_{2}$ vs. $\mathrm{P}_{2}$.

3. Binary hydrogen compounds In Figure 16.8, many binary hydrogen compounds, mainly elements in the center of the period table and the noble elements, are either uncharacterized or unknown. Why is this the case? How would you try to create these binary hydrogen compounds? Do you expect them to be saline, metallic, molecular, or perhaps none of these?

4. Ionic oxides Explain why metallic elements with low ionization energies commonly form basic ionic oxides and why nonmetal elements with high ionization energies form acidic oxides.

5. Size of the hydride ion Why is the hydride ion so large?

6. Alkali oxides Why does the principal product of the reaction of the alkali metals with oxygen vary the way it does $\left(\mathrm{Li}_{2} \mathrm{O}\right.$ to $\mathrm{Na}_{2} \mathrm{O}_{2}$ to $\left.\mathrm{KO}_{2}\right)$ ?

7. Boiling point trends Look at the boiling points of Group 2 elements in Table 16.4. Explain the trend.

8. Flame colors Explain why the alkaline earth metals burn with the colors they do (see the end of Section 16.7).

9. Nanostructured materials How do you expect the nano-/micro-structure of chemical and electroplated metals to differ? How about AC vs. DC electroplated metals? 
10. Stacked 2D materials Not only are $2 \mathrm{D}$ materials useful, but so are stacks of them, e.g. a graphene sheet stacked on top of an hBN sheet stacked on top of a graphene sheet. How might the properties of these stacked $2 \mathrm{D}$ materials differ from individual sheets? What might they be useful for? Stacked $2 \mathrm{D}$ materials is an area of active research from both experimental and theoretical perspectives.

11. Molecular motors Molecular motors rotate in a specified direction rather than clockwise or counterclockwise at random. How does this work?

12. Arsenic-based life Bacteria in high-temperature springs have been found to use arsenic instead of phosphorus in its DNA. Why can't these bacteria use phosphorus?

13. Phosphorus reactivity Explain why white phosphorus is more reactive than red phosphorus.

14. Sulfur chains Why is sulfur able to form long chains (catenate)?

15. Noble gases Use the melting and boiling points of the noble gases to estimate the energies of their London dispersion forces.

\section{Inquiries}

1. Chemical informatics The text motivates the importance of this and the next couple of chapters that are largely descriptive in nature by stating that this knowledge will help you develop chemical intuition. While this level of chemical intuition will get you far for exams, it would be of little use when tackling cutting edge research problems. After all, if we already had intuition for these problems, then we wouldn't be solving them! So let's start thinking about how data science can help us chemists develop intution. In Section 16.1, the textbook states that the five atomic properties principally responsible for the characteristic properties of each element are atomic radius, ionization energy, electron affinity, electronegativity, and polarizability. Many of these properties correlate with each other, however; for instance, elements with high electron affinity typically have high electronegativity. Run a principal component analysis (PCA) to reduce the number of descriptors necessary to describe the characteristic properties of each element. Based purely on these results, would you draw the periodic table differently?

2. More chemical informatics A more open-ended and difficult challenge than the PCA problem above is the solubility challenge proposed by Llinas et al.. Here's the basic idea: predicting the solubility of molecules is notoriously difficult yet of crucial importance for drug design, so Llinas et al. present accurately measured solubilities of 100 molecules and ask you to predict the solubility of 32. Start with multi-linear least squares regression and principal components analysis. Note that there are some issues with the training set. You can use the python libraries 'rdkit' for chemical informatics (importing chemical structures, for instance), 'pandas' for basic data analysis, and 'sklearn' for easy-to-use machine learning algorithms. Reports involving coding can be nicely presented with Jupyter Notebook. The authors have posted the results of the solubility challenge a year and ten years after issuing the challenge.

3. IVR Recent research has delved into understanding why chlorophyll is such an efficient transducer of sunlight to chemical energy, and one of the open questions is how the energy is absorbed at the head of the chlorophyll but output from the tail. We can tell that the text is a little outdated because it states that the $\mathrm{Mg}^{2+}$ helps to keep the ring rigid so that the incoming photon is not lost as heat, but if the molecule is totally rigid, then the energy would 
not be routed toward the tail where it is eventually turned into chemical energy. The study of how energy moves around in molecules is intramolecular vibrational redistribution (IVR). Ideally what must the vibrational structure of chlorophyll be to efficiently absorb photons at the head and convert it to chemical energy at the tail?

4. Silicon-based life Why do you think life is carbon-, not silicon-based? It's not an issue of limited resources given that the earth's crust contains so much silicon.

5. Buckminsterfullerene Conjecture what "shrink-wrapped" atoms (atoms in buckminsterfullerene molecules) might be useful for and how the properties would differ between atoms.

6. $2 \mathrm{D}$ materials There are thousands of $2 \mathrm{D}$ materials out there-the conductive graphene is just the beginning. We've talked a bit about hBN, for instance, which is insulating. An important class of $2 \mathrm{D}$ materials is dichalcogenides, such as $\mathrm{MoS}_{2}$, which are semiconductors. How might semiconducting $2 \mathrm{D}$ materials be useful?

Concluding notes Many properties of molecules and complexes can be explained with material from this textbook, but the effectiveness of this intuition fades in solids, where properties are dominated less by the properties of the elements comprising it than by the crystal structure and emergent effects accompanying it. See Ashcroft and Mermin, the standard solid state physics textbook, to learn more. For a more approachable introduction, see Oxford Solid State Basics by Steven Simon.

\section{THE ELEMENTS: THE D-BLOCK}

\section{Quick Takes}

1. Lanthanide or transition metal? Why might La and Ac be considered 'standard' transition metals and not f-block elements? Support your answer with quantitative evidence (i.e. look up their values of relevant properties compared to other f-block elements and transition metals).

2. Building-up principle The first paragraph of Section 17.1 states that "[a]ccording to the rules of the building-up principle, [the (n-1)] orbitals are the last orbitals to occupied . . . However, once they are occupied, they lie slightly lower in energy than the other ns-orbitals." This is naively contradictory, since if we are looking to find the ground state configuration, one would assume we would first occupy orbitals that lie lower in energy. Resolve this paradox.

3. Repulsion of d-electrons The textbook states that "[f]arther along the [dmetal] block, there are so many d-electrons that electron-electron repulsion increases more rapidly than the attraction from the nuclear charge, and the radii begin to increase again." Notice in Figure 17.2 that the atomic radii of the elements start increasing right at Fe. Explain why atomic radii start increasing right there. Explain whether the data is more likely to be from the pure gaseous or solid phase. Is it always true that in alloys, salts, and complexes containing these transition metals that the radii will starting increasing at Fe rather than another metal in this d-block series? Why or why not?

4. Oxidation states Explain the two patterns in oxidation states in Section 17.2: 1) Elements close to the center of each row have the widest range of oxidation states, and 2) elements in the second and third rows of the blocks are more likely to reach higher oxidation states than are those in the first row. 
5. Piezoelectric elements $\mathrm{BaTiO}_{3}$ is piezoelectric, meaning that it becomes electrically polarized when it is mechanically distorted. Name as many related compounds as you can that you expect to be piezoelectric, and compare against real data. Is it possible for an elemental solid to be piezoelectric? Does dimensionality (i.e. quantum dot vs. nanowire vs. $2 \mathrm{D}$ sheet vs. $3 \mathrm{D}$ solid) matter? Predict some piezomagnetic compounds.

6. Coinage metals Group $11^{\prime}$ s coinage metals have $(n-1) d^{10} n s^{1}$ valence electron configurations as opposed to $(n-1) d^{9} n s^{2}$. How would you experimentally determine this?

7. Solubility of halide salts Give a hand-wavey explanantion for why $\mathrm{AgNO}_{3}$ is soluble, AgF is sparingly soluble, and the other halide salts are not.

8. Mercury ions Give a hand-wavey explanation for why $\mathrm{Hg}_{2}^{2+}$ forms as opposed to $\mathrm{Hg}^{+}$.

9. 4 ligands Explain why $d^{8}$ compounds form square-planar complexes over tetrahedral complexes.

10. Chiral molecules Explain why chiral molecules rotate plane-polarized light from an EM perspective. What would happen to light with pure circular polarization?

11. Origin of chirality If it takes chiral reagents to synthesize chiral products to use as reagents for synthesizing other chiral products, and so on and so forth, how did chiral reagents appear in the first place in the universe? Think of a way to separate a racemic mixture into its enantiomers without using other chiral compounds.

12. Crystal field splitting energy Let's put some order of magnitude numbers to the effects of crystal field theory. The text states that "[t]ypically, the difference in energies of electrons in the $t_{2 g}$ - and the $e_{g}$-orbitals accounts only for about $10 \%$ of that energy, but it plays a major role in the optical and magnetic properties of the complex. How much is "that energy" and thus $10 \%$ of it? What are the energy regimes of relevant optical and magnetic properties? When would crystal field effects matter or not? This question is intentionally very open, so choose a couple properties that you're most interested in.

13. Spin polarization In Figure 17.29 and Figure 17.30, the orbital energy diagrams have 5 orbitals that can each take 2 electrons each with different spins. What happens to the orbital energy diagram if we turn on a magnetic field? Hint: you'll have to draw the diagram with spin-polarized orbitals, which is a fancy way of saying we split each original orbital that can hold 2 electrons into two orbitals, one of which can an up-spin while the other can carry a down-spin. How strong of a magnetic field would we need to start occupying $e_{g}$ orbitals before all $t_{2 g}$ orbitals have been filled?

14. Splitting energies of 4 ligand complexes Give an estimate for the difference in ligand-field splitting between octahedral and tetrahedral complexes.

15. Spectrochemical series Give a hand-wavey explanation to convince yourself of the ordering the spectrochemical series. Does the ordering correspond to the acidity? Size?

16. Absorptive transitions Why are charge-transfer transitions more intense than d-d transitions? (Hint: think about selection rules.)

17. Ligand field theory While ligand field theory is a rather old idea at this point, modern chemists continue to think about complexes through this theoretical lens, although of course the calculations to generate the level structures from 
first principles are done with computers and not by hand. Make sure you understand Section 17.12 deeply. What are some assumptions that ligand field theory holds? What are their implications, and when would they break down?

\section{Inquiries}

1. f-block elements Elements in the f-block have similar reactivity because the f-electrons are so deeply buried, resulting in the notorious difficulty of separating them from each other in ores and thus the high costs of their pure salts. (Compare, for example, the costs of lanthanide salts to more salts of more common elements.) What other properties do you expect f-block elements to differ in and that you could take advantage of to separate them from each other?

2. Spintronics What do you expect some pros and cons of spintronics vs. electronics to be?

Concluding notes Naming compounds is annoying. You should learn the basic rules here, but don't stress too much about knowing the intricate details. There are plenty of sites and software packages that will do it for you, such as

http://www. chemspider. com/StructureSearch.aspx. 